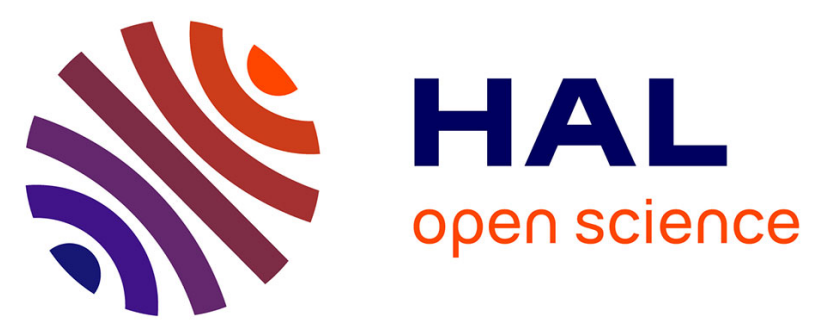

\title{
La fatigue au cours de la spondyloarthrite axiale débutante. Résultats de la cohorte française DESIR
}

Laure Gossec, Maxime Dougados, Maria-Antonietta d'Agostino, Bruno Fautrel

\section{- To cite this version:}

Laure Gossec, Maxime Dougados, Maria-Antonietta d'Agostino, Bruno Fautrel. La fatigue au cours de la spondyloarthrite axiale débutante. Résultats de la cohorte française DESIR. Revue du Rhumatisme, 2017, 10.1016/j.rhum.2017.05.008 . hal-01527226

\section{HAL Id: hal-01527226 https: / hal.sorbonne-universite.fr/hal-01527226}

Submitted on 24 May 2017

HAL is a multi-disciplinary open access archive for the deposit and dissemination of scientific research documents, whether they are published or not. The documents may come from teaching and research institutions in France or abroad, or from public or private research centers.
L'archive ouverte pluridisciplinaire HAL, est destinée au dépôt et à la diffusion de documents scientifiques de niveau recherche, publiés ou non, émanant des établissements d'enseignement et de recherche français ou étrangers, des laboratoires publics ou privés. 
La fatigue au cours de la spondyloarthrite axiale débutante. Résultats de la cohorte française DESIR

Laure Gossec $^{1,2}$, Maxime Dougados ${ }^{3,4}$, Maria-Antonietta D’Agostino ${ }^{5,6,7}$, Bruno Fautrel ${ }^{1,2}$

1: Institut Pierre Louis d'Epidémiologie et de Santé Publique, GRC-UPMC 08 (EEMOIS) ; Sorbonne Universités, UPMC Univ Paris 06, 75013

2 : Service de Rhumatologie, Hôpital Pitié Salpêtrière, AP-HP, 47-83 Bd de l'Hôpital, 75013 Paris, France

3 : Service de Rhumatologie, Hôpital Cochin, Université Paris Descartes, AP-HP ; 75014 Paris, France

4 : INSERM (U1153) : Epidémiologie clinique et biostatistiques, PRES Sorbonne Paris-Cité, 75013 Paris, France.

5: Faculté de médecine Simone Veil, Université de Versailles-Saint-Quentin-en-Yvelines, 78000 Versailles, France ;

6: INSERM U1173, Laboratoire d'Excellence INFLAMEX, 78180 Montigny-leBretonneux, France ;

7 : Service de Rhumatologie, Hôpital Ambroise Paré, Assistance Publique-Hôpitaux de Paris, 92100 Boulogne-Billancourt, France

Auteur correspondant: Laure Gossec, Hôpital Pitié-Salpêtrière, Service de Rhumatologie, 47-83, boulevard de l'Hôpital - 75013 Paris France

laure.gossec@aphp.fr

Tel: $+\underline{33142178421}$ Fax : +33142177959 


\section{Résumé}

Introduction: La fatigue est fréquente au cours de la spondyloarthrite axiale (axSpA) établie. La fréquence et les causes de la fatigue dans l'axSpA récente ne sont pas clairement identifiées. L'objectif de cette étude était d'évaluer l'intensité de la fatigue dans l'axSpA récente et d'évaluer si la fatigue était associée aux critères liés au patient ou liés à la maladie.

Méthodes : Les patients de DESIR, une cohorte observationnelle nationale de patients souffrant de douleurs inflammatoires dorsales récentes (<3 ans), évocatrices d'axSpA ont été analysés. L'intensité de la fatigue était évaluée selon une échelle numérique (0-10) à l'inclusion et sur un an. Les facteurs associés à une fatigue d'intensité élevée (> = 5/10) à 12 mois étaient évalués par régression logistique univariée et multivariée, chez les patients répondant aux critères ASAS de l'axSpA.

Résultats : Sur les 708 patients, 486 répondaient aux critères de l'ASAS pour l'axSpA : âge moyen $33 \pm 9$ ans ; 244 (50\%) hommes. L'intensité de la fatigue était élevée au départ (fatigue moyenne $5,7 \pm 2,3$ ) et ne diminuait que légèrement au cours de la première année. Elle concernait les patients répondant ou non aux critères ASAS. La fatigue intense à 12 mois était expliquée (variance expliquée 0,84) par un ASDAS-CRP élevé (odds ratio, OR, 3.05 [intervalle de confiance $95 \%, \mathrm{IC}, 2.27$ à 4.12], p < 0,0001), le BAS-G (OR 3,51 [IC, 2.8 à 6.2], $p<0,0001)$ et enfin, avec une signification limite, le sexe féminin $(O R, 1,65$ [IC, 1,00 à 2,71], $p$ $=0,049)$.

Conclusion : L'intensité de la fatigue était élevée dans cette population, pour les patients répondant ou non aux critères ASAS de l'axSpA. La fatigue intense était étroitement liée à certains paramètres de la maladie, suggérant qu'elle constitue une partie intégrante du processus pathologique au cours de l'axSpA récente.

Mots-clés : spondylarthrite / spondylarthrite ankylosante, fatigue, qualité de vie, BASDAI. 


\section{Introduction}

La fatigue est un symptôme important chez les patients atteints de maladies chroniques, notamment les rhumatismes, et a récemment été étudiée dans la polyarthrite rhumatoïde (PR) et dans l'arthrite psoriasique (PsA) [1-3]. La fatigue est une expérience subjective qui peut être décrite comme écrasante, comme un épuisement dysfonctionnel sans rapport avec l'effort, et mal dissipée avec un repos approprié [4-5]. La fatigue des patients atteints de rhumatismes influence leur qualité de vie et peut avoir des conséquences économiques importantes pour la société [6]. Dans les spondyloarthrites axiales (axSpA) établies, la fatigue semble être fréquente et 50 à $65 \%$ des patients la signalent [7-12]. La fatigue est d'ailleurs reconnue comme un marqueur de l'axSpA et est incluse dans le calcul de score BASDAI, largement utilisé pour évaluer l'activité de la maladie [13].

L’intensité de la fatigue au début d'une axSpA n'a toutefois pas été caractérisée. Les niveaux de fatigue pourraient être différents au cours des stades précoces car l'ankylose rachidienne n'est généralement pas encore présente et la qualité de vie pourrait en être moins altérée. Les causes de la fatigue dans les rhumatismes inflammatoires tels que l'axSpA ou la PR ne sont pas claires. La fatigue liée à l'axSpA est décrite de diverses façons, soit comme étant liée aux caractéristiques intrinsèques des patients, par exemple les facteurs démographiques, psychologiques et sociaux, soit aux caractéristiques de la maladie, par exemple son activité. [10, 12, 14-15].

Ainsi, bien que la fatigue soit fréquemment signalée et importante dans la SpA, sa cause reste incertaine et sa présence au début de la SpA et dans les différents sous-groupes de SpA (forme axiale prédominante ou non, avec ou sans sacro-iliite radiographique) a très peu été étudiée [12]. 
Les objectifs de cette étude étaient d'évaluer l'intensité de la fatigue au début la SpA (et dans les différents sous-types de $\mathrm{SpA}$ ) et d'évaluer si la fatigue était plus fortement associée au patient ou aux caractéristiques de la maladie.

\section{Méthodes}

\section{Protocole d'étude et patients}

La cohorte DESIR [16] est prospective, multicentrique, observationnelle et inclut des patients souffrant de douleurs inflammatoires suggérant une axSpA datant de moins de 3 ans. Les traitements sont libres dans DESIR et laissés au choix du rhumatologue traitant.

\section{Évaluation de la fatigue}

La fatigue était évaluée selon une échelle de notation numérique (ENN) de 0 à 10 au cours de la première année de suivi (soit à l'inclusion dans la cohorte, puis à 6 et 12 mois), en utilisant la première question du BASDAI : «Comment décririez-vous le niveau global de votre fatigue / fatigabilité au cours de la dernière semaine?" [13]. Une fatigue intense était définie par un score $>=5 / 10$ (comme décrit par d'autres auteurs) $[10,11]$.

\section{Facteurs associés à la fatigue}

L'association entre des niveaux de fatigue élevés à 12 mois, et d'autres facteurs était analysée. Les facteurs évalués étaient (a) les caractéristiques relatives aux patients à l'inclusion: variables démographiques (âge, sexe, durée des symptômes, origine ethnique), statut familial (marié ou non, nombre d'enfants) et les années d'étude comme statut socioéconomique; (b) les caractéristiques liées à l'activité de la maladie à 12 mois : les variables d'activité de la maladie, score d'activité de la maladie (ASAS : ASDAS-CRP [17], score global de Bath pour la spondylarthrite ankylosante (BAS-G), et le questionnaire d'évaluation de capacité fonctionnelle HAQ adapté à l'AxSpA : HAQ-AS), et (c) d'autres caractéristiques de la maladie (HLA-B27, sous-type de la maladie selon le médecin, signes extra-articulaires oui / 
non, douleur enthésique oui / non évaluée par le score d'enthésite de Maastricht et sacroiliite selon les critères de New York de spondylarthrite ankylosante, par radiographies ou imagerie par résonance magnétique, avec interprétation locale de l'image.

\section{Analyses statistiques}

L'intensité de la fatigue était évaluée dans l'ensemble de la population par des statistiques descriptives à l'inclusion, après 6 mois et après un an, chez tous les patients de la cohorte DESIR puis dans les sous-groupes répondant ou non au départ aux critères d'évaluation de la spondylarthrite (ASAS) pour l'axSpA [18] et ses différentes variantes, notamment les sousgroupes ASAS clinique et ASAS imagerie, ou selon les sous-types de la maladie (purement axiale ou axiale + périphérique ou indifférenciée, tel que définis par les enquêteurs).

Après un an de suivi : une régression logistique multivariée puis univariée était réalisée pour expliquer la fatigue élevée, définie par l'EN de fatigue $>=5 / 10$ à 12 mois, par les variables explicatives à travers une sélection progressive. Le score BASDAI n'était pas pris en compte dans les analyses puisque la question de la fatigue était une partie intégrante de ce score et que les corrélations avec ASDAS-CRP étaient élevées. L'indice fonctionnel de Bath pour la spondylarthrite ankylosante n'était également pas analysé car fortement corrélé à I'HAQ-AS. Cette analyse a été réalisée dans le groupe de patients répondant aux critères ASAS, et après exclusion du BAS-G car l'évaluation globale des patients et la fatigue sont souvent corrélées. II n'y avait aucune imputation de données manquantes. Le Statistical Analysis System (SAS) de la version 9.2 était utilisé.

\section{Rôle de la source de financement :}

Pour la cohorte DESIR, une subvention sans restrictions de Wyeth Pharmaceuticals a été allouée pour les 5 premières années du suivi des patients recrutés dans la cohorte DESIR.

Pour la présente étude : ce travail a été soutenu par Pfizer grâce à une subvention sans restriction (Passerelle).

Divulgations d'intérêts : non applicable à ce travail. 


\section{Résultats}

\section{Caractéristiques des patients}

Parmi les 708 patients de la cohorte DESIR (moyenne d'âge \pm déviation standard $34 \pm 9$ ans ; 327 (46 \%) des hommes), 486 répondaient aux critères de l'ASAS pour une axSpA au départ (âge moyen $33 \pm 9$ ans ; 244 (50\%) des hommes). La durée des symptômes était courte et le BASDAI était élevé à l'inclusion dans la cohorte (tableau 1) ; 206 (30,1\%) avaient des taux élevés de protéine C-réactive. Bien que tous les patients présentaient des symptômes axiaux, les investigateurs ont distingué maladie axiale $(N=349)$, axiale et périphérique $(\mathrm{N}=41)$, indifférenciée $(\mathrm{N}=225)$ ou autre / pas clair $(\mathrm{N}=93)$. Après un an de suivi, 450 (72,9\%) patients étaient traités par anti-inflammatoires non stéroïdiens, et 158 $(22,3 \%)$ recevaient des anti-TNF.

L’intensité de la fatigue était élevée au départ (fatigue moyenne, 5,7 $\pm 2,3$ ). Chez les patients répondant aux critères ASAS ou non, des différences numériques étaient relevées (fatigue 5,5 $\pm 2,4$ et 6,0 $\pm 2,2$ respectivement) ; cependant il y avait un chevauchement important entre les 2 groupes; les valeurs médianes de la fatigue étaient identiques dans les deux groupes (6 [intervalle interquartile 4-7] et 6 [5-8], respectivement) (tableau 2). II y avait cependant des différences selon les sous-catégories de critères ASAS : la fatigue était de 4,7 $\pm 2,5$ dans le groupe sacroiliite évaluée par radiographie, le groupe spondylarthrite ankylosante $(N=50), 5,6$ \pm 2,3 dans le groupe sacroiliite évaluée par IRM (sans sacroiliite radiographique) $(\mathrm{N}=98)$, contre 5,9 $\pm 2,2$ dans le groupe non-radiographique $(N=197)$ ( $p=0,0009$ par rapport au groupe spondylarthrite ankylosante).

Les niveaux de fatigue selon le sous-type de la maladie étaient similaires (dans les maladies à prédominance axiale, fatigue $5.6 \pm 2.4$, dans les formes périphériques $6.0 \pm 2.2$ et dans les 
formes indifférenciées $5,7 \pm 2,3$, respectivement; $p=0,6$ ). La fatigue diminuait de manière significative, mais faible, lors de la première année de suivi (moyenne de fatigue à un an, 4,9 \pm 2,6, $p=0,008$ ) (tableau 2). Ces baisses étaient similaires à la diminution de la douleur (données non présentées).

\section{Facteurs associés à des niveaux élevés de fatigue à un an}

Parmi les 428 patients répondant aux critères ASAS pour l'axSpA et avec des données complètes disponibles pour cette analyse, un niveau de fatigue élevé $(>=5 / 10)$ était relevé chez 210 (49\%) patients à 12 mois. En analyse univariée, la fatigue était associée au sexe féminin ( $p<0,0001)$, un ASDAS-CRP plus élevé $(p<0,0001)$, un BAS-G supérieur ( $p$ $<0,0001$ ), un HAQ-AS supérieur ( $p<0,0001)$, une plus courte scolarité $(p=0,003$ ) et une négativité pour le HLA B27 ( $p=0,026)$. L'analyse multivariée révélait une fatigue élevée à 12 mois expliquée (variance expliquée, 0,84) par les éléments indépendants suivants (tableau 3) : ASDAS-CRP plus élevé (odd Ratio, OR, 3.05 [95\% intervalle de confiance, IC, 02/27 au 04/12], $\mathrm{p}<0,0001)$, BAS-G plus élevé (OR, 3,51 [IC à $95 \%, 2.8$ à 6.2], $p<0,0001)$ et, avec une signification limite, sexe féminin (OR, 1,65 [IC 95\%, 1,00 à 2,71], $p=0,049$ ). Les caractéristiques liées au patient, outre le sexe, n'étaient pas significatives pour expliquer la fatigue à 12 mois dans cette analyse multivariée. En excluant le BAS-G de l'analyse, les facteurs explicatifs restaient liés à la maladie (variance expliquée, 0,82; tableau 3).

Dans l'ensemble de la population, la fatigue élevée était expliquée par le ASDAS-CRP (OR, 3,12 [IC $95 \%, 02 / 31$ au 04/20], p < 0,0001), le BAS-G (OR, 2,42 [IC $95 \%, 1,53$ à 3,84], p $=0,00021$ ) et le HAQ-AS (OR pour une augmentation de 0,2, 1,15 [IC à $95 \%, 1.3$ à 1.30], p $=0,017)$ (variance expliquée, 0,84).

\section{Discussion}

Les niveaux de fatigue étaient élevés dans cette grande cohorte de patients avec des symptômes évocateurs de début d'axSpA, indépendamment des critères ASAS. La fatigue 
intense après un an était étroitement liée à l'activité de la maladie plutôt qu'aux variables liées au patient. Ceci suggère que la fatigue fait partie intégrante de l'activité des phases précoces de l'axSpA.

Notre étude est la première à notre connaissance à explorer la fatigue et les facteurs associés à l'axSpA précoce. Du fait d'un cadre de références personnel différent, les symptômes tels que la fatigue pourraient être très différents en début de maladie ; néanmoins les niveaux de fatigue relevés ici étaient similaires à ceux observés dans l'axSpA établie.

Cette étude comporte des points forts et des points faibles. Le nombre d'échantillons étudiés était élevé, et représente un ensemble en France de patients atteints de rachialgies inflammatoires suggérant une axSpA [16]. La fatigue pouvant néanmoins être influencée par le contexte culturel [19], des études dans d'autres pays sont nécessaires. Le questionnaire de BASDAI sur la fatigue a été utilisé pour évaluer la fatigue, plutôt qu'une échelle de fatigue spécifique comme l'échelle Functional Assessment of Chronic IIIness Therapy (FACIT-F). Cependant, des corrélations satisfaisantes ont été obtenues entre les ENN de fatigue et des échelles plus complexes [10, 20]. Enfin, certains éléments importants tels que les troubles du sommeil et l'état psychologique y compris l'anxiété et dépression n'ont pas été pris en compte dans la présente étude.

La fatigue est un symptôme fréquent dans les maladies rhumatismales, avec près de la moitié des patients décrivant des niveaux de fatigue élevés, dans la PR ainsi que dans le rhumatisme psoriasique ou l'axSpA [2-4, 10]. Dans l'axSpA établie, les niveaux de fatigue sont très similaires à ceux observés dans la population actuelle en début de la maladie [10,12]. Les niveaux de fatigue dans l'axSpA devraient toutefois être replacés en contexte général, en utilisant la population saine comme témoin car la fatigue est souvent vécue par les adultes en bonne santé avec une prévalence comprise entre 14 et $25 \%$ et une prédominance féminine [11]. 
Dans la présente étude, une légère diminution des niveaux de fatigue était relevée après l'inclusion dans la cohorte, mais après un an, une fatigue > = 5/10 existait chez encore $55 \%$ des patients. La diminution de la fatigue au cours de la première année de suivi dans DESIR est peut-être liée aux traitements reçus, ou à un effet de régression vers la moyenne. Des observations similaires ont été faites dans des cohortes de PR précoces [21]. Dans la cohorte actuelle, l'utilisation de thérapies anti-TNF concernait une minorité de patients au cours de la première année de suivi, et n'a pas été spécifiquement analysée ici. Les niveaux de fatigue élevés observés dans l'axSpA et les conséquences potentielles de cette fatigue sont étayés par peu de publications sur ce sujet: une revue systématique de la littérature indique seulement 3 articles sur la fatigue dans la SpA sur une période de 10 ans en Europe, contre 16 papiers dans la PR pour la même période [22].

Nos résultats indiquent des niveaux de fatigue similaires dans la population de patients présentant des symptômes évocateurs d'axSpA, que les patients remplissent ou non les critères ASAS pour l'axSpA. Ceci indique que le critère de fatigue ne devrait pas être utilisé comme élément de diagnostic en cas de douleur inflammatoire suggérant une axSpA. Les patients présentant une axSpA non radiographique avaient des niveaux plus élevés de fatigue que les patients évalués par radiographie, mais il y avait un chevauchement important entre ces populations ainsi que les patients ne répondant pas aux critères de classification de l'axSpA [12]. La question de la fibromyalgie concomitante chez les patients atteints axSpA non radiographique pourrait être soulevée ici.

La fatigue est difficile à traiter pour les rhumatologues. Tout d'abord, parce que, comme nous l'avons montré ici, la fatigue ne semble pas être utile pour le diagnostic et la classification. De plus, la fatigue n'est pas toujours un marqueur de l'activité de la maladie et ne conduit ainsi pas toujours à des changements de traitements de fond; et enfin son traitement, comme symptôme isolé, est difficile. 
Dans cette étude, la fatigue était étroitement liée à l'activité de la maladie plutôt qu'à des variables liées au patient. Le sexe féminin semble être associé à une fatigue plus élevée, à la fois dans la population générale [11], et l'axSpA établie [12]. II a été rapporté que la fatigue au cours de l'axSpA établie était liée à des facteurs démographiques, psychologiques et sociaux, mais aussi à l'activité de la maladie. [10, 12, 14-15, 23]. Nous avons mis en évidence, comme d'autres auteurs, que la fatigue était fortement associée à d'autres paramètres rapportés par les patients au cours de l'évaluation globale du patient et de sa qualité de vie [10,12] : Ceci est l'une des difficultés des études sur la fatigue évaluée selon des propriétés psychométriques, mais ceci indique également l'importance potentielle de la fatigue du point de vue du patient [24].

Pour essayer de faire la distinction entre les causes liées à la maladie et les symptômes rapportés par les patients dans la recherche des origines de la fatigue, nous avons effectué des analyses en incluant puis en excluant, l'évaluation globale du patient (BAS-G), et nous avons utilisé le score ASDAS-CRP comme marqueur de l'activité de la maladie. II en résultait que les facteurs associés étaient principalement liés à la maladie plutôt qu'au patient. Nous pourrions ainsi émettre l'hypothèse que la fatigue est plus étroitement liée à l'évolution de la maladie dans les SpA que dans la PR. La fatigue n'apparait néanmoins pas prise en compte dans le score ASDAS [17] suggérant une faible valeur prédictive, au moins dans la maladie établie.

À ce jour, il n'y a pas de traitement ciblant spécifiquement la fatigue dans l'axSpA. En effet, les mécanismes sous-jacents de la fatigue dans l'axSpA restent mal compris, et pourraient par exemple impliquer les cytokines pro-inflammatoires et le processus inflammatoire, et / ou la détresse psychologique. Une étude récente basée sur l'imagerie par résonance magnétique 3T du cerveau a indiqué que la fatigue au cours de l'axSpA peut impliquer l'acuité sensorielle et les réseaux d'attention cérébraux [19]. Quoi qu'il en soit, l'efficacité des traitements habituels prescrits par les rhumatologues pour la fatigue, y compris les traitements ciblés, est seulement 
modérée dans la PR [25]. Dans cette dernière maladie, de même, l'efficacité des interventions non pharmacologiques telles que les thérapies cognitivo-comportementales a été démontrée ; et ces interventions restent à évaluer dans l'axSpA [26].

\section{Remerciements}

La cohorte DESIR dépend de l'Assistance Publique-Hôpitaux de Paris via I'Unité de recherche clinique Paris-Centre et sous l'égide de la Société Française de Rhumatologie et de I'INSERM (Institut National de la Santé et de la Recherche Médicale). La gestion de la base de données est effectuée dans le département d'épidémiologie et de biostatistique (Professeur Jean-Pierre DAURES, D.I.M., Nîmes, France).

Nous tenons à remercier les différents centres régionaux participants: $\operatorname{Pr}$ Maxime Dougados (Paris - Cochin B), Pr André Kahan (Paris - Cochin A), Pr Olivier Meyer (Paris Bichat), Pr Pierre Bourgeois (Paris - La Pitié-Salpetrière), Pr Francis Berenbaum (Paris - Saint Antoine), Pr Pascal Claudepierre (Créteil), Pr Maxime Breban (Boulogne Billancourt), Dr Bernadette Saint-Marcoux (Aulnay-sous-Bois), Pr Philippe Goupille (Tours), Pr Jean-Francis Maillefert (Dijon), Dr Xavier Puéchal (Le Mans), Pr Daniel Wendling (Besançon), Pr Bernard Combe (Montpellier), Pr Liana Euller-Ziegler (Nice), Pr Philippe Orcel (Paris - Lariboisière), Pr Pierre Lafforgue (Marseille), Dr Patrick Boumier (Amiens), Pr Jean-Michel Ristori (ClermontFerrand), Dr Nadia Mehsen (Bordeaux), Pr Damien Loeuille (Nancy), Pr René-Marc Flipo (Lille), Pr Alain Saraux (Brest), Pr Corinne Miceli (Le Kremlin Bicêtre), Pr Alain Cantagrel (Toulouse), Pr Olivier Vittecoq (Rouen).

Nous tenons également à remercier Simon Paternotte, statisticien, auparavant de l'hôpital Cochin, Paris et Adrien Etcheto, statisticien, Hôpital Cochin de Paris pour l'aide statistique. 


\section{Références}

1. Jason LA, Evans M, Brown M, Porter N. What is fatigue? Pathological and non pathological fatigue. PM R $2010 ; 2(5): 327-31$.

2. Kirwan JR, Minnock P, Adebajo A, et al. Patient perspective : fatigue as a recommended patient centered outcome measure in rheumatoid arthritis. J Rheumatol. 2007 ;34(5):1174-7.

3. Husted JA, Tom BD, Schentag CT, Farewell VT, Gladman DD.. Occurrence and correlates of fatigue in psoriatic arthritis. Ann Rheum Dis 2009 ; 68: 1553-58.

4. Hewlett S, Cockshott Z, Byron M, et al. Patients' perceptions of fatigue in rheumatoid arthritis : overwhelming, uncontrollable, ignored. Arthritis Rheum. 2005 ;53(5):697-702.

5. Günaydin R., Göksel Karatepe A., Çeşmeli N., Kaya T. Fatigue in patients with ankylosing spondylitis : relationships with disease-specific variables, depression, and sleep disturbance. Clin Rheumatol 2009 ; 28:1045-1051.

6. Carlotto A, Hogsett VL, Maiorini EM, Razulis JG, Sonis ST. The economic burden of toxicities associated with cancer treatment : review of the literature and analysis of nausea and vomiting, diarrhoea, oral mucositis and fatigue. Pharmacoeconomics. 2013 ;31(9):753-66.

7. Ibn Yacoub Y, Amine B, Laatiris A, Abouqal R, Hajjaj-Hassouni N. Assessment of fatigue in Moroccan patients with ankylosing spondylitis. Clin Rheumatol $2010 ; 29: 1295-1299$.

8. Da Costa D, Dritsa M, Ring A, Fitzcharles MA. Mental Health status and leisure-time physical activity contribute to fatigue intensity in patients with spondylarthropathy. Arthritis Rheum $2004 ; 51:$ 1004-1008.

9. Turan Y, Duruöz M, Bal S, Guvenc A, Cerrahoglu L, Gurgan A. Assessment of fatigue in patients with ankylosing spondylitis. Rheumatol Int 2007 ; 27:847-852.

10. Van Tubergen A, Coenen J, Landewe R, et al. Assessment of fatigue in patients with ankylosing spondylitis : a psychometric analysis. Arthritis Rheum 2002 ; 47:8-16. 
11. Dagfinrud H, Vollestad N, Loge J, Kvien T, Mengshoel AM. Fatigue in patients with Ankylosing Spondylitis: A comparison With the general population and associations With Clinical and Self-Reported Measures. Arthritis Rheum 2005 ; 53: 5-11.

12. Bianchi WA, Elias FR, Carneiro S, et al. Assessment of fatigue in a large series of 1492 Brazilian patients with Spondyloarthritis. Mod Rheumatol. 2014 ;24(6):980-4.

13. Garrett S, Jenkinson T, Kennedy LG, Whitelock H, Gaisford P, Calin A. A new approach to defining disease status in ankylosing spondylitis : the Bath Ankylosing Spondylitis Disease Activity Index. J Rheumatol. 1994 ;21:2286-91.

14. Dernis-Labous E, Messow M, Dougados M. Assessment of fatigue in the management of patients with ankylosingspondylitis. Rheumatology 2003 ; 42:1523-1528.

15. Calin A, Edmuns L, Kennedy LG. Fatigue in ankylosing spondylitis-Why is it ignored? J Rheumatol 1993 ;20: 991-5.

16. Dougados M, d'Agostino MA, Benessiano J, et al. The DESIR cohort : a 10-year followup of early inflammatory back pain in France : study design and baseline characteristics of the 708 recruited patients. Joint Bone Spine. $2011 ; 78: 598-603$

17. Machado P, Landewé R, Lie E, et al. Ankylosing Spondylitis Disease Activity Score (ASDAS) : defining cut-off values for disease activity states and improvement scores. Ann Rheum Dis. $2011 ; 70(1): 47-53$.

18. Rudwaleit M, van der Heijde D, Landewé R, et al. The development of Assessment of SpondyloArthritis international Society classification criteria for axial spondyloarthritis (part II) : validation and final selection. Ann Rheum Dis. 2009 ;68(6):777-83.

19. Hifinger M, Putrik $P$, Ramiro $S$, et al. In addition to individual demographic and clinical measures, levels of fatigue are dependent on country of residence. An analysis among 3920 patients from 17 countries (the COMORA study). Ann Rheum Dis 2014 ;73(Suppl2) : 411.

20. Hewlett S, Dures E, Almeida C. Measures of fatigue : Bristol Rheumatoid Arthritis Fatigue Multi-Dimensional Questionnaire (BRAF MDQ), Bristol Rheumatoid Arthritis Fatigue 
Numerical Rating Scales (BRAF NRS) for severity, effect, and coping, Chalder Fatigue Questionnaire (CFQ), Checklist Individual Strength (CIS20R and CIS8R), Fatigue Severity Scale (FSS), Functional Assessment Chronic Illness Therapy (Fatigue) (FACIT-F), MultiDimensional Assessment of Fatigue (MAF), Multi-Dimensional Fatigue Inventory (MFI), Pediatric Quality Of Life (PedsQL) Multi-Dimensional Fatigue Scale, Profile of Fatigue (ProF), Short Form 36 Vitality Subscale (SF-36 VT), and Visual Analog Scales (VAS). Arthritis Care Res (Hoboken). 2011 ;63 Suppl 11 :S263-86.

21. Rat AC, Pouchot J, Fautrel B, Boumier P, Goupille P, Guillemin F. Factors associated with fatigue in early arthritis : results from a multicenter national French cohort study. Arthritis Care Res (Hoboken). 2012 ;64(7):1061-9.

22. Gossec L, Berenbaum F, Chauvin P, et al. Reporting of patient-perceived impact of rheumatoid arthritis and axial spondyloarthritis over 10 years : a systematic literature review. Rheumatology (Oxford). 2014 ;53(7):1274-81.

23. Chauffier K, Paternotte S, Burki V, et al. Fatigue in spondyloarthritis : a marker of disease activity. A cross-sectional study of 266 patients. Clin Exp Rheumatol. 2013 ;31(6):86470.

24. Kiltz U, van der Heijde D, Boonen A, et al. Development of a health index in patients with ankylosing spondylitis (ASAS HI) : final result of a global initiative based on the ICF guided by ASAS. Ann Rheum Dis. $2015 ; 74(5): 830-5$

25. Chauffier K, Salliot C, Berenbaum F, Sellam J. Effect of biotherapies on fatigue in rheumatoid arthritis : a systematic review of the literature and meta-analysis. Rheumatology (Oxford). $2012 ; 51(1): 60-8$.

26. Hewlett S, Ambler N, Almeida C, et al. Self-management of fatigue in rheumatoid arthritis : a randomised controlled trial of group cognitive-behavioural therapy. Ann Rheum Dis. $2011 ; 70(6): 1060-7$. 
Tableau 1. Caractéristiques des patients de la cohorte DESIR à l'inclusion

\begin{tabular}{|c|c|c|c|}
\hline Caractéristiques à l'inclusion & $\begin{array}{l}\text { Population } \\
\text { globale } \\
\quad(\mathrm{N}=708)\end{array}$ & $\begin{array}{c}\text { Critères } \\
\text { ASAS remplis } \\
(\mathrm{N}=486)\end{array}$ & $\begin{array}{l}\text { Critères } \\
\text { ASAS non } \\
\text { remplis } \\
\qquad(\mathrm{N}=206)\end{array}$ \\
\hline Age, ans, moyenne (DS) & $33.7(8.6)$ & $33.0(8.6)$ & $35.4(8.4)$ \\
\hline hommes, $\mathrm{N}(\%)$ & $327(46.2)$ & $244(50.2)$ & $76(36.9)$ \\
\hline $\begin{array}{l}\text { Durée des symptômes à l'inclusion } \\
\text { (mois), moyenne (DS) }\end{array}$ & $18.1(10.4)$ & $18.5(10.6)$ & $17.2(10.0)$ \\
\hline Positivité HLA B27, N (\%)* & $410(58.0)$ & $406(83.7)$ & $3(1.5)$ \\
\hline sacro-iliite radiographique, $\mathrm{N}(\%)^{*}$ & $187(27.0)$ & $187(38.9)$ & 0 \\
\hline synovite périphérique, $\mathrm{N}(\%)$ & $51(7.2)$ & $35(7.2)$ & $13(6.3)$ \\
\hline Douleur enthésique, $\mathrm{N}(\%)$ & $347(49.0)$ & $227(46.7)$ & $114(55.3)$ \\
\hline $\begin{array}{l}\text { Manifestations extra-articulaires à } \\
\text { l'inclusion, N (\%) }\end{array}$ & $188(26.6)$ & $132(27.2)$ & $53(25.7)$ \\
\hline BADSAI (0-100), moyenne (DS) & $44.7(20.0)$ & $42.9(20.4)$ & $48.4(18.4)$ \\
\hline ASDAS-CRP, moyenne (DS) & $2.5(1.0)$ & $2.5(1.1)$ & $2.5(0.9)$ \\
\hline $\begin{array}{l}\text { Douleur axiale EEN (0-10), moyenne } \\
\text { (DS) }\end{array}$ & $5.0(2.7)$ & $4.7(2.8)$ & $5.6(2.4)$ \\
\hline BASFI (0-100), moyenne (DS) & $30.4( \pm 22.8)$ & $29.7( \pm 22.5)$ & $32.3( \pm 23.5)$ \\
\hline BAS-G (0-10), moyenne (DS) & $5.1(2.6)$ & $4.9(2.6)$ & $5.6(2.3)$ \\
\hline HAQ-AS (0-3), moyenne (DS) & $0.6( \pm 0.5)$ & $0.6( \pm 0.5)$ & $0.6( \pm 0.5)$ \\
\hline
\end{tabular}

Pour 16 patients $(2,2 \%)$, les critères de classification ASAS ne pouvaient pas être calculés en raison de données manquantes.

DS : déviation standard, EEN : échelle d'évaluation numérique, BASDAI: Bath spondylarthrite ankylosante Disease Activity Index [13], ASDAS-CRP : score d'activité ASAS 
CRP [17], BASFI : index fonctionnel de Bath pour la spondylarthrite ankylosante, BAS-G : BAS global, HAQ-AS : Questionnaire de santé adapté à l'axSpA.

* Les pourcentages indiqués sont ceux obtenus à partir des données disponibles 
Tableau 2. Intensité de la fatigue sur un an dans la cohorte DESIR

\begin{tabular}{|c|c|c|c|c|}
\hline $\begin{array}{l}\text { Fatigue EEN (0-10), } \\
\text { moyenne DS) }\end{array}$ & $\begin{array}{l}\text { Population } \\
\text { globale } \\
\qquad(\mathrm{N}=708)\end{array}$ & $\begin{array}{c}\text { Critères } \\
\text { ASAS remplis } \\
(\mathrm{N}=486)\end{array}$ & $\begin{array}{l}\text { Critères ASAS } \\
\text { non remplis } \\
(\mathrm{N}=206)\end{array}$ & $\begin{array}{l}\text { Valeur } \\
\text { de } P^{*}\end{array}$ \\
\hline A l'inclusion & $5.7(2.3)$ & $5.5(2.4)$ & $6.0(2.2)$ & 0.009 \\
\hline Après 6 mois & $4.9(2.6)$ & $4.7(2.6)$ & $5.5(2.5)$ & 0.001 \\
\hline Après 12 mois & $4.9(2.6)$ & $4.7(2.6)$ & $5.5(2.4)$ & 0.001 \\
\hline
\end{tabular}

classification ASAS pour la SpA à l'inclusion [18] 
Tableau 3. Facteurs associés à une forte fatigue à 12 mois de suivi dans la cohorte DESIR.

Niveau bas de fatigue» $(\mathrm{N}=211)$ modèle complet

Odds ratio [intervalle de confiance de 95 \%] (valeur p) Modèle hors BAS-G

Odds ratio [95 \% intervalle de confiance] (valeur p)

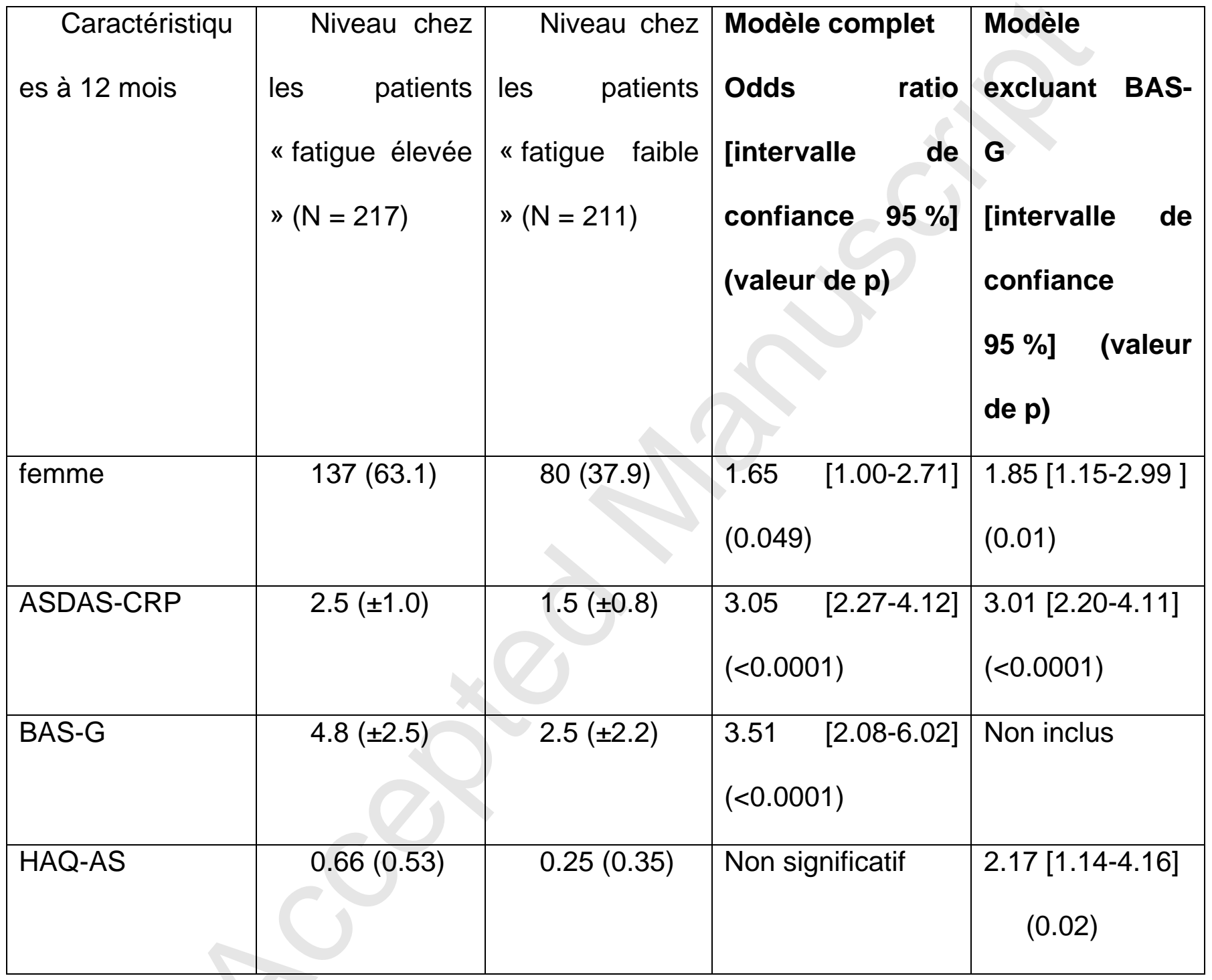

Régression logistique multivariée de 428 patients répondant aux critères de classification ASAS pour l'axSpA et avec des données complètes disponibles. Les niveaux de chaque variable dans les groupes fatigue élevée et fatigue faible sont donnés à titre indicatif, comme $\mathrm{N}$ (\%) ou moyenne (écart-type). Les odds ratios étaient calculés pour une augmentation de 1,0 de I'ASDAS-CRP, une augmentation de 5 unités pour le BAS-G et une augmentation de 0,2 points du HAQ-AS. 